\title{
Development and Application of Remote Monitoring System for Distribution in Rural Areas
}

\author{
Hongjun Ju \\ Lijiang Teachers College, Lijiang, Yunnan, 674100, China
}

Keywords: Rural Distribution, Remote Monitoring System, Development and Application.

\begin{abstract}
In the process of rural development and construction, distribution network construction is a very important content, and through the application of distribution remote monitoring system can better improve the construction process of distribution network, improve the quality of construction. A remote monitoring system for rural distribution operation, which can remotely test leakage protector and monitor the running condition of distribution station area, is developed. Nowadays, there are still problems such as frequent tripping of leakage protector and difficulty of manual transmission in rural distribution network system. This paper mainly analyzes the development and application of rural distribution remote monitoring system, discusses the main development process and specific application of the monitoring system, hoping to play some reference role for the relevant staff.
\end{abstract}

\section{Development of Remote Monitoring System for Distribution Rural Areas}

With the deepening of rural power management system, the personnel of rural power supply stations have been reduced compared with the past, but their scope and difficulty and workload have increased obviously. Especially after the cancellation of the mode of one village and one electrician, if the leakage protector of rural power distribution station area appears tripping phenomenon, it will not be able to effectively guarantee the reliability of power supply and the quantity of electricity sold in rural areas. After this situation, the personnel of the power supply station need to travel to and from each distribution station area continuously, and there will be problems such as power transmission difficulties. In order to solve these problems effectively, in addition to establishing a perfect duty system, the remote monitoring system of distribution should be developed through the application of network and electronic technology. The system has the functions of reflecting the running state of AC contactor, the action of leakage protector and the oil temperature alarm of transformer. In the process of rural distribution network construction, remote control system can also be used to realize the remote operation of AC contactors, the phase-dividing test of leakage protector and the remote control of test delivery. In this way, it can effectively solve the related problems in the rural distribution process, relieve the working pressure of power supply personnel, reduce the workload, and improve the quality of distribution construction in rural areas[1].

\subsection{Developing Ideas}

The development of distribution remote monitoring system should include two links, first of all, to the remote release of leakage protector memory latch test device development, so as to effectively alleviate the current leakage protector after memory latchup problems. Second, specific monitoring systems should be developed. In the process of system development, we should adhere to the principles of economy and practicality, and consider the future distribution management system so that it can effectively connect with the distribution automation system.[2]. 


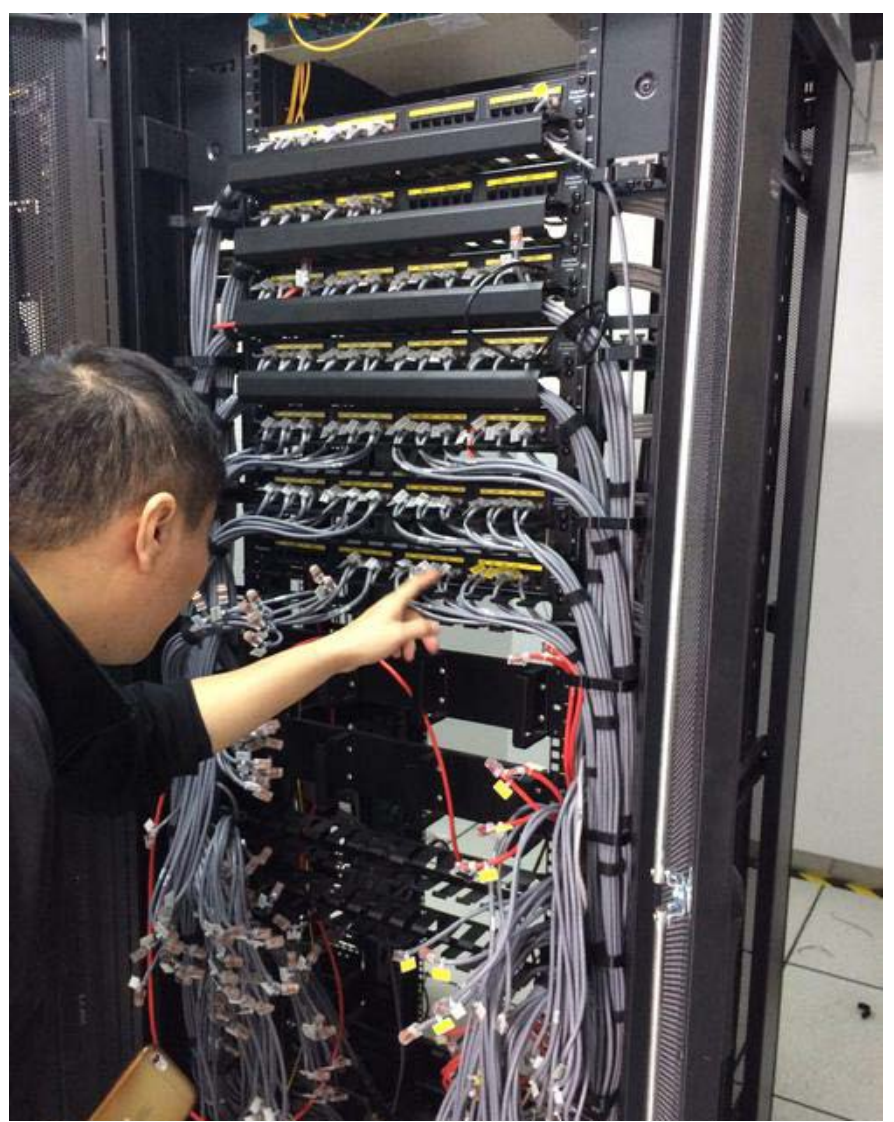

Figure 1 Distribution remote monitoring system

\subsection{Main Working Principles and Specific Functions of the Remote Monitoring System}

At the distribution stations in rural areas, the leakage protector is tripped due to the leakage of electricity from the line or equipment, and when the memory is locked, the user mainly informs the power supply station by telephone, and the latter sends the relevant signal to the missing device after receiving the signal from the BP machine in the test transmitter, which can drive the relevant circuit of the test transmitter, so that the power supply of the leakage protector is disconnected and the memory lock is released. In this way, power transmission can be re-transmitted to the power failure line, and if the relevant leakage fault is recovered during the process, it will be successful, and if the power failure means that the leakage fault has not been eliminated, through this way can effectively achieve the purpose of trial transmission[3].

When the following cases occur, the monitoring device will have the corresponding information feedback, first of all, when the distribution station area related lines or equipment leakage problems will make the leakage protector open, and it will be transmitted to the monitoring device corresponding change signal. Second, when the prepaid electricity user is enabled, there will be information feedback when the amount of electricity remaining is smaller than that of the latter. When the current, voltage, oil temperature and so on of the distribution network do not match the set data, and the monitoring device will appear information feedback when the relevant data is higher. The signal of the monitoring device can be transmitted through the telecommunication network or wireless communication network, while the monitoring device can respond to the change signal with specific graphic and text changes or voice prompts, so as to effectively monitor the operation of the distribution station area. The operation control of the monitoring device is simple, when the leakage protector appears tripping and memory locking, it needs to stop and send electricity to the station area, and the relevant duty personnel can send specific stop and send electrical signals to their monitoring device through software. After receiving the signal, the leakage protector will be switched on or cut off, which will effectively control the coil power of the contactor, so as to effectively realize the memory of the leakage protector lock test or stop transmission and other related purposes, and through the monitoring system can also carry out the 
partial grounding test of the leakage protector[4].

\section{Application of Remote Monitoring System Rural Distribution}

\subsection{Actual Application}

This paper analyzes the remote monitoring system of rural distribution operation in a certain area. After a period of research and development, the first monitoring device in the area has successfully produced the system management software, and has been installed and tested in many locations in the area, and has achieved success. In the course of operation of the system, the system has been improved digitally by the relevant researchers, drawing on the opinions of all parties. At present, the system has been effectively applied in dozens of distribution stations here, and has a very remarkable effect[5].

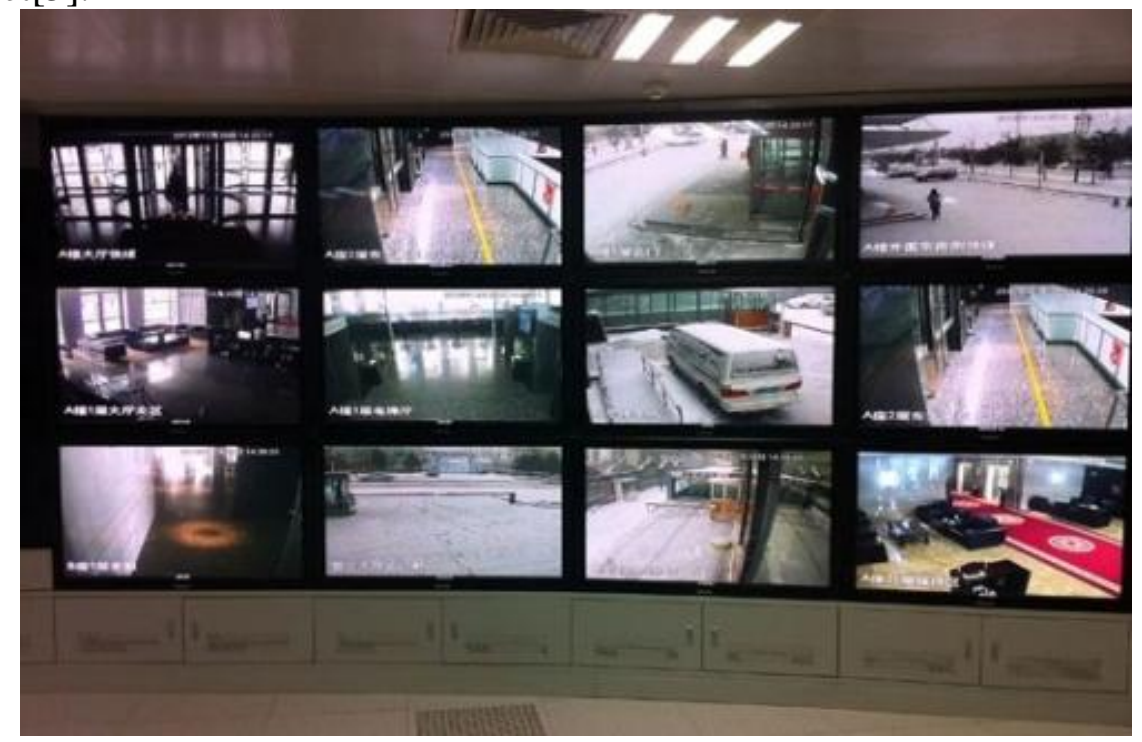

Figure 2 Remote monitoring system

\subsection{Features of Remote Monitoring System}

low cost and has great promotion potential

For the rural distribution remote monitoring system development and application costs are relatively low, and in the application process can also achieve very obvious results, and can effectively solve the problems in the process of distribution construction, so it has great potential for promotion.

\subsection{Perfect Function With Strong Practicability}

The remote monitoring system can monitor the distribution operation condition, and can also take the remote way to remove the memory locking of the leakage protector, which has the related functions of remote meter reading and line damage analysis, especially the remote release function of the memory locking of the leakage protector can effectively solve the practical problems existing in the power supply process in rural areas after the reform of the system and improve the power supply quality in an all-round way.

\subsection{Simple Maintenance and Stable Performance}

The remote monitoring system is very simple and convenient in the installation process, it can be installed on the fitting device, and its wiring process is not complicated, some people who have knowledge of electrical knowledge can master the specific installation method after learning. And the operation of the monitoring system only requires personnel after a brief study, so that they can master the relevant operational skills, and do not need to be related to computer professional knowledge training. Through the relevant statistics, after the remote monitoring device is installed, the correct action and the correct action rate are high, and the data obtained are also very reliable. 


\subsection{Use Effect is Remarkable}

First of all, the application of remote monitoring system can achieve very obvious economic benefits, in the leakage protector maintenance and management costs can be effectively reduced, but also can reduce the related expenses of the personnel responsible for power transmission. After using this system, the success rate of trial delivery has been obviously improved, so that the sales of each power supply has also been significantly increased, thus effectively improving the economic benefits of power supply enterprises.

Secondly, the remote monitoring system also has the function of remote meter reading and line damage analysis, so it can effectively help the power supply station to carry out line loss management, which also creates considerable economic benefits. After installing the remote monitoring system, we can effectively carry out the clue management through the functions of remote meter reading and line loss automatic analysis, which can reduce the economic loss of tens of thousands of yuan for the power supply department in this area, which greatly improves the economic benefit of the power supply station[6].

Finally, the application of the system can also achieve very obvious social benefits. After the application of the system in the local power supply station, the reliability of the power supply in the distribution station area has been obviously improved, and the satisfaction degree of the users has become higher, and the system has been unanimously praised. This has also facilitated the charging of power supply stations, and the related staff and workers believe that the remote monitoring system can effectively help them to carry out power distribution management, and the workload and the degree of hard work of power transmission between the power supply stations at night have also improved significantly. Through the application of remote monitoring system, the labor productivity of power supply management has been obviously improved, which also effectively improves the social benefits of power supply.

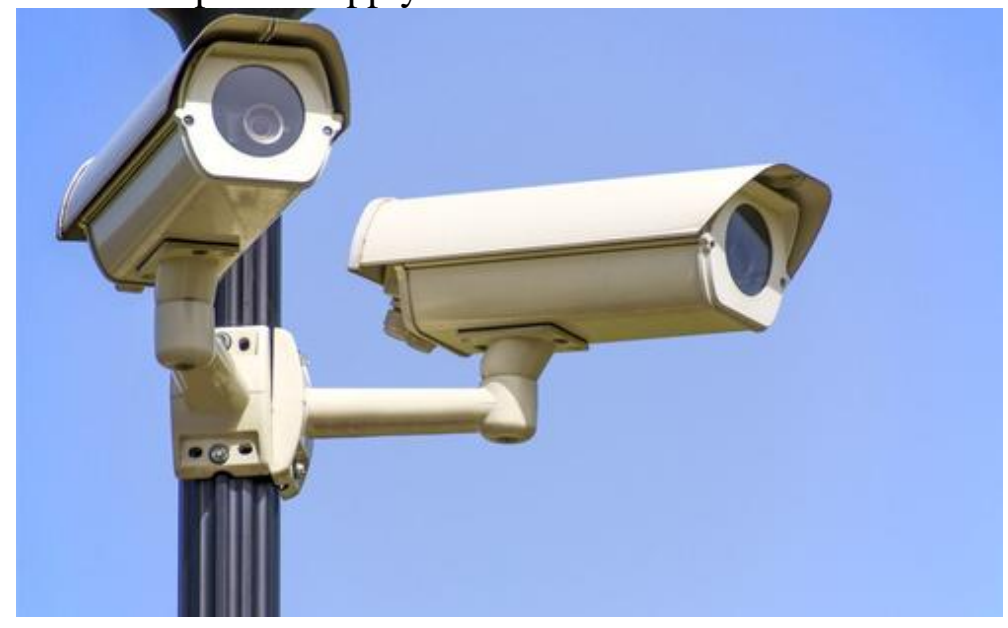

Figure 3 Monitoring

\section{Conclusion}

To sum up, the application of distribution remote monitoring system in rural areas can better enhance the economic benefits of power supply enterprises, and for the development of society, it can set up a good social image for the relevant power supply enterprises, thus promoting the stable development of power supply industry in China. And through the application of the system can effectively enhance the rural distribution network technology content, so that the distribution network more safe and reliable, to meet the demand for electricity. In the future, with the development of science and technology, the related functions of remote monitoring system will be further upgraded and perfected, so that it can play a better role in the construction of rural distribution network. 


\section{References}

[1] Li, Chao. A Study on Power Detection and Remote Control Technology in Distribution Interval. Changjiang University, 2019.

[2] Yang, Yang., Lai, Chunyao., Qin, Qi., Shan., Hewei, Yang, Zihang., long, Zhensheng. Application of Intelligent Remote Monitoring Terminal in Split Air Conditioning Distribution Centralized Control System. Electric World , vol. 58, no. 11, pp. 43-49, 2017.

[3] Yellowstone. Research on Economic Operation Technology of Intelligent Power Supply and Distribution System. China University of Geosciences (Beijing).

[4] Guo, Yanjie., Ma, Jing., Wang, Jiguang., Guo, Yintan. Development and application of remote monitoring and management system for rural variable frequency water supply equipment. Shandong Water Conservancy, no. 08, pp. 44-45, 2017.

[5] Hu, Yongqin., Yi, Songhua. Development and Application of Rural Distribution Remote Monitoring System. Hunan Electric Power, no. 06, pp. 59-60, 2017.

[6] Hu, Yongqin. Development and Application of Remote Monitoring System for Rural Distribution Operation. Chinese Society of Surveying and Mapping. Building a well-off society in an all-round way: the historical responsibility of Chinese science and technology workers Proceedings of the 2016 academic annual meeting of the Chinese Association of Science and Technology (top). China Society of Surveying and Mapping: China Society of Surveying and Mapping, no. 331, 2016. 